\title{
Similarity Solution of (2+1)-Dimensional Calogero-Bogoyavlenskii-Schiff Equation Lax Pair
}

\author{
Shaimaa Salem ${ }^{1}$, Magda Kassem², Samah Mohamed Mabrouk ${ }^{2, *}$ \\ ${ }^{1}$ Department of Mathematics and Physics, Faculty of Engineering, Higher Technological Institute, $10^{\text {th }}$ of Ramadan City, Egypt \\ ${ }^{2}$ Department of Mathematics and Physics, Faculty of Engineering, Zagazig University, Zagazig, Egypt
}

Email address:

eng.shema@gmail.com (S. Salem),mkassem@live.com (M. Kassem), samahmm@gmail.com (S. M. Mabrouk)

${ }^{*}$ Corresponding author

\section{To cite this article:}

Shaimaa Salem, Magda Kassem, Samah Mohamed Mabrouk. Similarity Solution of (2+1)-Dimensional Calogero-Bogoyavlenskii-Schiff Equation Lax Pair. American Journal of Applied Mathematics. Special Issue: Analytical Approaches to Nonlinear Science and Applications. Vol. 7, No. 5, 2019, pp. 137-144. doi: 10.11648/j.ajam.20190705.11

Received: September 10, 2019; Accepted: September 23, 2019; Published: October 12, 2019

\begin{abstract}
In this paper, we discussed and studied the solutions of the (2+1)-dimensional Calogero-Bogoyavlenskii-Schiff (CBS) equation. The Calogero-Bogoyavlenskii-Schiff equation describes the propagation of Riemann waves along the y-axis, with long wave propagating along the x-axis. Lax pair and Bäcklund transformation of the Calogero-Bogoyavlenskii-Schiff equation are derived by using the singular manifold method (SMM). The optimal Lie infinitesimals of the Lax pair are obtained. The detected Lie infinitesimals contain eight unknown functions. These functions are optimized through the commutator table. The eight unknown functions are evaluated through the solution of a set of linear differential equations, in which solutions lead to optimal Lie vectors. The CBS Lax pair is reduced by using the optimal Lie vectors to a system of ordinary differential equations (ODEs). The solitary wave solutions of Calogero-Bogoyavlenskii-Schiff equation Lax pair's show soliton and kink waves. The obtained similarity solutions are plotted for different arbitrary functions and compared with previous analytical solutions. The comparison shows that we derive new solutions of Calogero-Bogoyavlenskii-Schiff equation by using the combination of two methods, which is different from the previous findings.
\end{abstract}

Keywords: Calogero-Bogoyavlenskii-Schiff Equation, Singular Manifold Method, Lax Pair, Lie Infinitesimals, Similarity Solutions

\section{Introduction}

Derivation of the Lax pairs of a nonlinear partial differential equation (NLPDE) needs first the study of its integrability, such as, the existence of a sufficiently large number of conservation laws or symmetries [1-4]. Many methods are used for studying the integrability of nonlinear partial differential equations. Among them the singular manifold method based on Painlevè analysis [5-7], homogeneous balance method [8-11], Weiss, Tabor and Carnevale (WTC) method [12], symbolic computation method [13] and Bäcklund transformation (BT) [14]. We here derive the Lax pair for Calogero-Bogoyavlenskii-Schiff (CBS) equation [15-19];

$$
u_{x t}+u_{x} u_{x y}+\frac{1}{2} u_{x x} u_{y}+\frac{1}{4} u_{x x x y}=0
$$

This equation describes the $(2+1)$ dimensional interaction of Riemann wave propagating along the y-axis with long wave propagating along the $\mathrm{x}$-axis [15-19]. CBS equation was investigated from various perspectives, such as the classical and non-classical methods. Through several symmetry reductions, exact solutions of the CBS equation were derived [20], while a variety of exact solutions using the improved $\left(\mathrm{G}^{\prime} / \mathrm{G}\right)$-expansion method were presented [21-23], the symbolic computation method [24, 25], the exponential expansion method [26], the improved tanh-coth method [27], the symmetry method [28], the Hirota's bilinear method to derive its multiple front solutions [29]. Here the singular manifold method is used to deduce the CBS Lax pair. Then we proceed to a similarity reduction of this Lax pair to a system of ordinary differential equations obtain optimal similarity solutions and compare our results with previous work on CBS equation. The organization of this paper is as follows: In Section 2 the Lax pair is deduced for CBS equation. In Section 
3 the similarity solutions for this Lax pair are deduced. Finally we present the conclusions in section 4 .

\section{Singular Manifolds Method}

In this section, the Singular Manifold Method is applied to find the BT and Lax pair for the $(2+1)$ dimensional CBS equation (1). Singular Manifold Method is an inverse solution [30-32] of nonlinear partial differential equations having a series form;

$$
u(x, y, t)=\sum_{j=0}^{\infty} u_{j}(x, y, t) \phi(x, y, t)^{j-\alpha}
$$

Where $\phi(x, y, t)$ is an Eigen function and $\alpha$ is a real number obtained from the dominant behavior analysis.

\subsection{Bäcklund Transformation of CBS Equation}

Replacing for (2) into (1), the dominant behavior analysis yields $\alpha=1$, in this case the series expansion (2) reduces to:

$$
u=u_{0} \varphi^{-1}+u_{1}
$$

This is the Bäcklund transformation of the Calogero-Bogoyavlenskii-Schiff equation. Substitute from (3) into (1), then equating the coefficients of the similar powers of $\varphi$ to zero yields;

Coefficient of $\varphi^{-4}$;

$$
u_{0}=2 \varphi_{x}
$$

Replacing for $\mathrm{u}_{0}$ in (3) reduces it to;

$$
u=\frac{2 \varphi_{x}}{\varphi}+\mathrm{u}_{1}=2(\ln \varphi)_{x}+u_{1}
$$

\subsection{Lax Pair of CBS Equation}

Equation (1) Lax pair's is deduced by substituting (5) into (1) and equating the coefficients of the similar powers of $\varphi$ to zero giving;

Coefficient of $\varphi^{-2}=0$;

$$
-4 u_{1 X} \varphi_{x y} \varphi_{x}-3 \varphi_{x} \varphi_{x x} u_{1 y}-2 u_{1 x} \varphi_{x x} \varphi_{y}-2 \varphi_{x}^{2} u_{1 x y}-4 \varphi_{x t} \varphi_{x}+\varphi_{x x y} \varphi_{x x}-2 \varphi_{x x x y} \varphi_{x}-u_{1 x x} \varphi_{x} \varphi_{y}-2 \varphi_{x x} \varphi_{t}-\frac{1}{2} \varphi_{x x x x} \varphi_{y}=0
$$

Coefficient of $\varphi^{-3}=0$;

$$
4 u_{1 x} \varphi_{x}^{2} \varphi_{y}+2 \varphi_{x}^{3} u_{1 y}+2 \varphi_{x}^{2} \varphi_{x x y}+4 \varphi_{x}^{2} \varphi_{t}+2 \varphi_{x x x} \varphi_{x} \varphi_{y}-2 \varphi_{x} \varphi_{x x} \varphi_{x y}-\varphi_{x x}^{2} \varphi_{y}=0
$$

Then defined new variables $V, R$ and $Z$ as follows;

$$
\mathrm{V}=\frac{\varphi_{x x}}{\varphi_{x}}, \mathrm{R}=\frac{\varphi_{t}}{\varphi_{x}} \text { and } \mathrm{Z}=\frac{\varphi_{y}}{\varphi_{x}}
$$

Substitute (8) into (6) and (7) leads to the two equations;

$$
\begin{gathered}
-6 u_{1 x} Z V-4 u_{1 X} Z_{X}-3 V u_{1 y}-2 u_{1 x y}-u_{1 x x} Z-\frac{7}{2} Z V V_{x}-6 R V-4 R_{x}+V V_{y}-V^{2} Z_{x}-\frac{3}{2} Z V^{3}-2 V_{x y}-4 V V_{y}-2 Z_{x} V_{x}-\frac{1}{2} Z V_{x x}=0 \\
-4 R=4 u_{1 x} Z+2 u_{1 y}+2 V_{y}+2 V_{x} Z+V^{2} Z
\end{gathered}
$$

Equations (9) and (10) can be easily linearized by introducing a new function $\psi$ defined as:

$$
\varphi_{x}=\psi^{2}
$$

By substituting (11) into (8) yields;

$$
\begin{gathered}
V=2 \frac{\psi_{x}}{\psi} \\
Z_{x}+Z V=2 \frac{\psi_{y}}{\psi} \\
R_{x}+R V=2 \frac{\psi_{t}}{\psi}
\end{gathered}
$$

Then, by substituting (12), (13) and (14) into (9) and (10) respectively, we get:

$$
\begin{gathered}
\left(-4 u_{1 x} \psi_{x}-u_{1 x x} \psi-\psi_{x x x}-3 \frac{\psi_{x} \psi_{x x}}{\psi}\right) Z-8 u_{1 x} \psi_{y}-6 u_{1 y} \psi_{x}-2 u_{1 x y} \psi-4 R \psi_{x}-8 \psi_{t}-4 \psi_{x x y}-4 \frac{\psi_{y} \psi_{x x}}{\psi}+4 \psi_{y} \psi_{x}^{2}=0 \\
-4 R=4 u_{1 x} Z+2 u_{1 y}+4 \frac{\psi_{x y}}{\psi}-4 \frac{\psi_{x} \psi_{y}}{\psi^{2}}+4 Z \frac{\psi_{x x}}{\psi}
\end{gathered}
$$

By replacing for (16) into (15) provides us with two equations;

$$
\begin{gathered}
-u_{1 x x} \psi-\psi_{x x x}+\frac{\psi_{x} \psi_{x x}}{\psi}=0 \\
-8 u_{1 x} \psi_{y}-4 u_{1 y} \psi_{x}-2 u_{1 x y} \psi-8 \psi_{t}-4 \psi_{x x y}-4 \frac{\psi_{y} \psi_{x y}}{\psi}+\frac{4 \psi_{y} \psi_{x x}}{\psi}=0
\end{gathered}
$$


Dividing (17) by $\psi$ and integrating with respect to ' $x$ ' leads to the first Calogero-Bogoyavlenskii Lax pair

$$
\psi_{x x}+\left(u_{1 x}-\lambda\right) \psi=0
$$

Where $\lambda$ is a constant of integration. Then setting $\psi_{x}=\psi_{y}$ in (18) we obtain the second Lax pair

$$
4 \psi_{t}+4 u_{1 x} \psi_{y}+2 u_{1 y} \psi_{x}+u_{1 x y} \psi+2 \psi_{x x y}=0
$$

\section{The Similarity Solutions of CBS Lax Pair}

\subsection{Lie Infinitesimals of CBS Equation}

The Lie infinitesimals of the CBS Lax pair (19) and (20) have the form;

$$
\begin{gathered}
V_{1}=f_{1}(t) \frac{\partial}{\partial x}+\frac{\partial}{\partial t}+\left[2 y f_{1 t}(t)+f_{2}(t)\right] \frac{\partial}{\partial u} \\
V_{2}=f_{3}(t) \frac{\partial}{\partial x}+\frac{\partial}{\partial y}+\left[2 y f_{3 t}(t)+f_{4}(t)\right] \frac{\partial}{\partial u} \\
V_{3}=f_{5}(t) \frac{\partial}{\partial x}+\psi \frac{\partial}{\partial \psi}+\left[2 y f_{5 t}(t)+f_{6}(t)\right] \frac{\partial}{\partial u} \\
V_{4}=\left[f_{7}(t)+\frac{x}{3}\right] \frac{\partial}{\partial x}+\frac{y}{3} \frac{\partial}{\partial y}+t \frac{\partial}{\partial t} \\
+\left[2 y f_{7 t}(t)+\frac{\lambda}{3}(2 x-4 y)-\frac{u}{3}+f_{8}(t)\right] \frac{\partial}{\partial u}
\end{gathered}
$$

The arbitrary functions $f_{i}(t), i=1.8$, are optimized through the commutative products listed in Table 1. This leads to a system of ordinary differential equations in the unknown functions $f_{i}(t)$ reported here;

Table 1. Commutator table.

\begin{tabular}{lllll}
\hline & $\mathbf{V}_{\mathbf{1}}$ & $\mathbf{V}_{\mathbf{2}}$ & $\mathbf{V}_{\mathbf{3}}$ & $\mathbf{V}_{\mathbf{4}}$ \\
\hline $\mathrm{V}_{1}$ & 0 & 0 & 0 & $\mathrm{~V}_{1}$ \\
$\mathrm{~V}_{2}$ & 0 & 0 & 0 & $\frac{V_{2}}{3}$ \\
$\mathrm{~V}_{3}$ & 0 & 0 & 0 & 0 \\
$\mathrm{~V}_{4}$ & $-\mathrm{V}_{1}$ & $\frac{-V_{2}}{3}$ & 0 & 0 \\
\hline
\end{tabular}

$$
\left\{\begin{array}{c}
f_{7 t}=\frac{2}{3} f_{1}+t f_{1 t}, f_{4 t}-2 f_{1 t}=0 \\
-\frac{2}{3} f_{4}-t f_{4 t}=\frac{4 \lambda}{3}-2 f_{7 t} \\
2 y f_{7 t t}+f_{8 t}=2 t f_{1 t t} y+\frac{10}{3} f_{1 t} y+\frac{4}{3} f_{2}+t f_{2 t}-\frac{2 \lambda}{3} f_{1} \\
f_{3 t}=0, f_{5 t}=0, f_{6 t}=0
\end{array}\right.
$$

Solving this system of ODE's (25), leads to the values of functions $f_{i}(t), i=1 \ldots 8$, listed below;

$$
\begin{gathered}
f_{1}(\mathrm{t})=\frac{\lambda}{3}, f_{2}(\mathrm{t})=1, f_{3}(\mathrm{t})=0, f_{4}(\mathrm{t})=\frac{-4 \lambda}{3}, f_{5}(\mathrm{t})=0, \\
f_{6}(\mathrm{t})=0, f_{7}(\mathrm{t})=\frac{2 \lambda}{9} \mathrm{t} \text { and } f_{8}(\mathrm{t})=\left(\frac{4}{3}-\frac{2 \lambda^{2}}{9}\right) \mathrm{t}
\end{gathered}
$$

According to these values the Lie vectors (21) to (24) is rewritten as:

$$
\begin{gathered}
V_{1}=\frac{\lambda}{3} \frac{\partial}{\partial x}+\frac{\partial}{\partial t}+\frac{\partial}{\partial u} \\
V_{2}=\frac{\partial}{\partial y}+\left[\frac{-4 \lambda}{3}\right] \frac{\partial}{\partial u} \\
V_{3}=\psi \frac{\partial}{\partial \psi} \\
V_{4}=\left[\frac{2 \lambda}{9} \mathrm{t}+\frac{x}{3}\right] \frac{\partial}{\partial x}+\frac{y}{3} \frac{\partial}{\partial y}+t \frac{\partial}{\partial t}+ \\
{\left[\frac{4 \lambda}{9} y+\frac{\lambda}{3}(2 x-4 y)-\frac{u}{3}+\left(\frac{4}{3}-\frac{2 \lambda^{2}}{9}\right) \mathrm{t}\right] \frac{\partial}{\partial u}}
\end{gathered}
$$

Vectors $\mathrm{V}_{1}$ to $\mathrm{V}_{4}$ are used to reduce and solve the Lax system (19) and (20).

Vector $V_{1}$ is used to reduce the system of equations (19) and (20), then solve it giving the following two solutions;

$$
\begin{gathered}
u_{1}=F_{1}(y)+t+\lambda x-\frac{1}{3} \lambda^{2} t \\
u_{2}=\frac{3 x+F_{2}\left(y, \frac{-3 x+\lambda t}{\lambda}\right) \lambda}{\lambda}
\end{gathered}
$$

Where $F_{1}$ is an arbitrary function of $y, F_{2}$ is an arbitrary function of $(y, t)$ and $\lambda$ is a constant of integration. These solutions are plotted for $=1, F_{1}=\frac{\sin y}{y}, F_{2}=\frac{e^{-y^{2}} \sin (t-3 x)}{(t-3 x)}$, in (Figure $1(\mathrm{a}, \mathrm{b})$ ) for $t=0.1$ and in (Figure $2(\mathrm{a}, \mathrm{b})$ ), for $\mathrm{t}=1$.

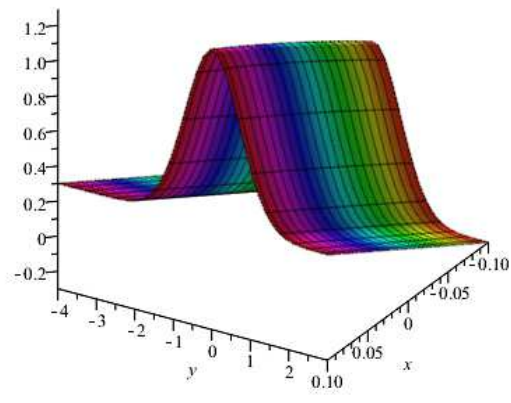

(a) $u_{1}(x, y, t)=3 x+\frac{e^{-y^{2}} \sin (0.1-3 x)}{(0.1-3 x)}$

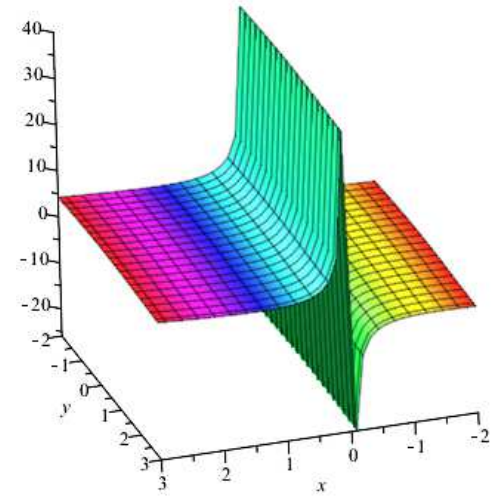

(b) $u_{2}(x, y, t)=\frac{2}{x}+\frac{\sin y}{y}+x+\frac{1}{15}$

Figure 1. Solutions of CBS equation for vector $V_{1}$ at time $t=0.1$ and $t=1$. 


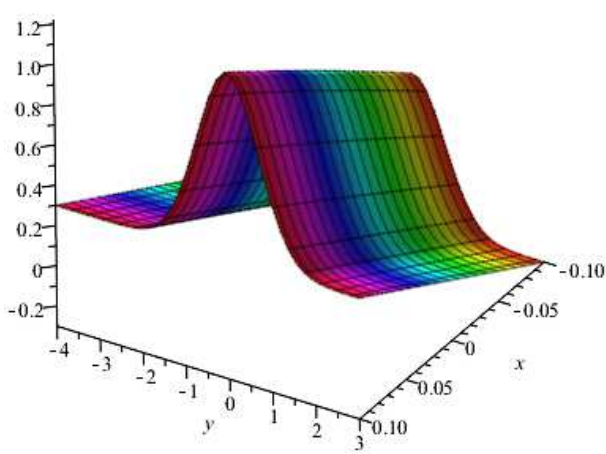

(a) $u_{3}(x, y, t)=3 x+\frac{e^{-y^{2}} \sin (1-3 x)}{(1-3 x)}$

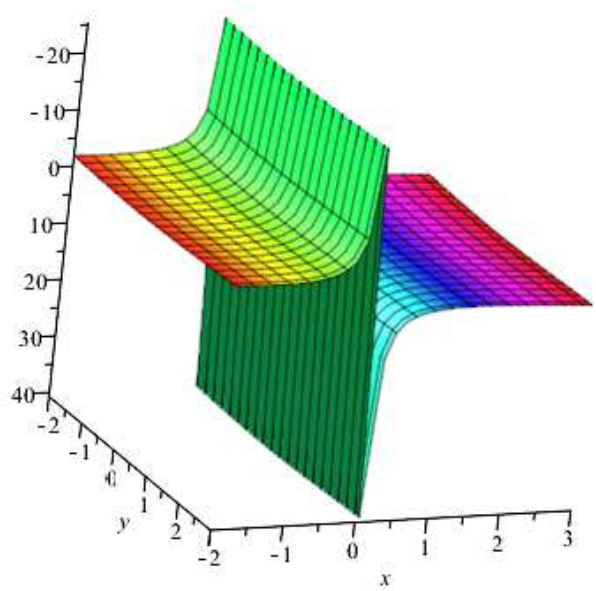

(b) $u_{4}(x, y, t)=\frac{2}{x}+\frac{\sin y}{y}+x+\frac{2}{3}$

Figure 2. Solutions of CBS equation for vector $V_{1}$ at time $t=0.1$ and $t=1$.

The solution of the Lax system (19) - (20) by using the vector $\mathrm{V}_{2}$ are;

$$
\begin{gathered}
u_{3}=\frac{-4}{3} \lambda y+F_{3}(x, t) \\
u_{4}=F_{4}(t)+\frac{1}{3}(-4 y+3 x) \lambda
\end{gathered}
$$

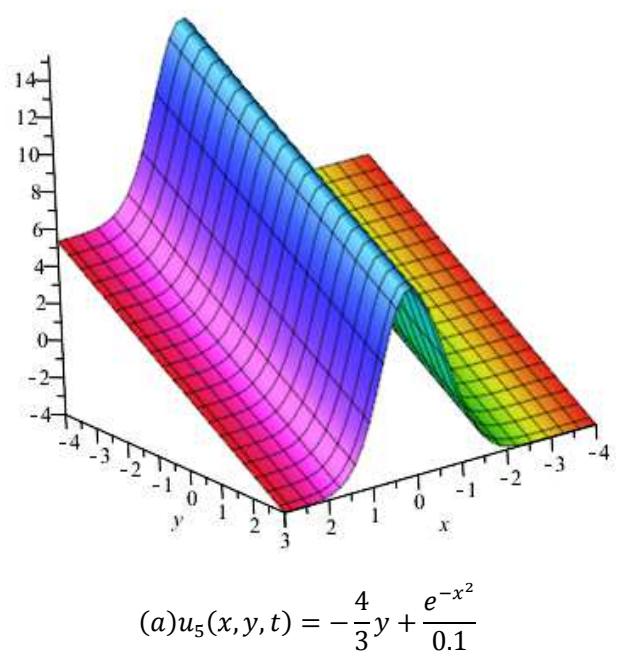

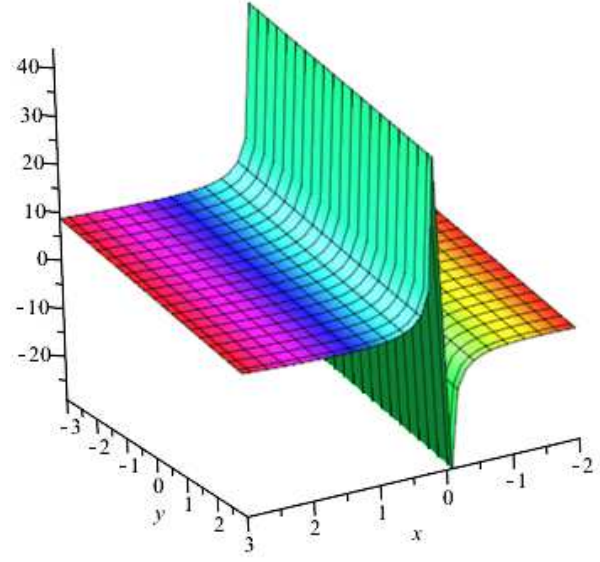

(b) $u_{6}(x, y, t)=\frac{2}{x}+\frac{\sin (0.1)}{0.1}+\frac{1}{3}(-4 y+3 x)$

Figure 3. Solutions of CBS equation for vector $V_{2}$ for $t=0.1$ and $t=1$.

Where $\mathrm{F}_{3}$ is an arbitrary function of $(x, t)$ and $\mathrm{F}_{4}$ is an arbitrary function of $(\mathrm{t})$. Choosing, $F_{3}=\frac{e^{-x^{2}}}{t}, F_{4}=\frac{\sin t}{t}$, the solutions (33) and (34) are plotted for $\lambda=1$ as depicted in (Figure 3(a,b)), for $t=0.1$ and in (Figure 4(a, b)), for $t=1$.

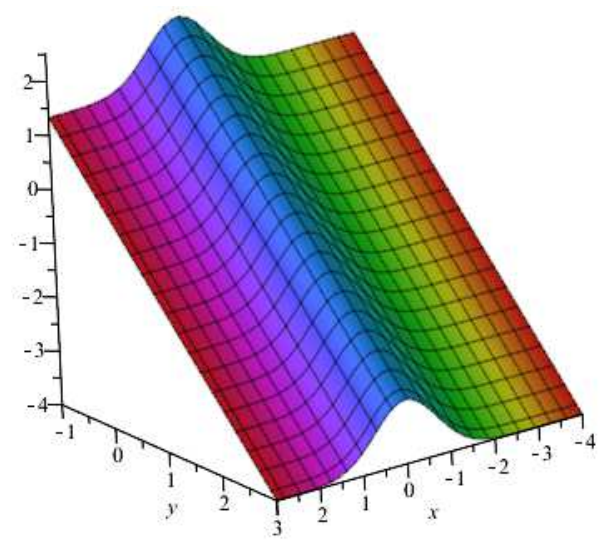

$$
\text { (a) } u_{7}(x, y, t)=-\frac{4}{3} y+\frac{e^{-x^{2}}}{1}
$$

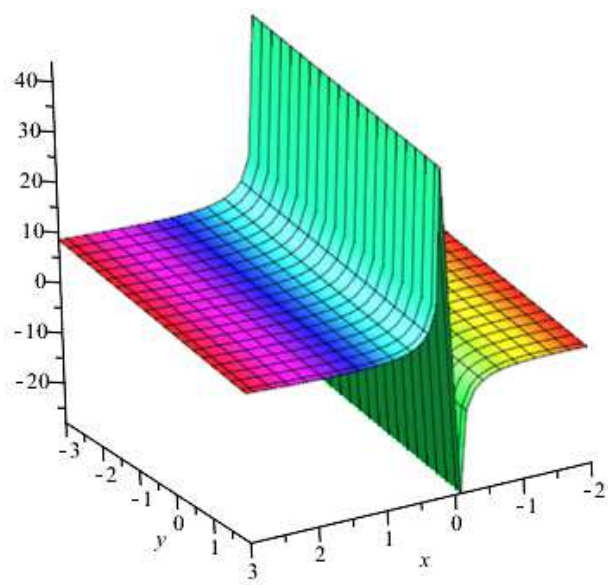

(b) $u_{8}(x, y, t)=\frac{2}{x}+\frac{\sin (1)}{1}+\frac{1}{3}(-4 y+3 x)$

Figure 4. Solutions of CBS equation for vector $V_{2}$ for $t=0.1$ and $t=1$. 


\subsection{Comparison with Previous Works}

We do then compare results obtained using vectors $V_{l}$ and $V_{2}$, (31)-(34) with previous solutions of $(2+1)$-dimensional CBS equation as in the following.

Bruzon and Gandarias [20] used the classical and non-classical symmetry methods to obtain symmetry reductions and exact solutions of the $(2+1)$-dimensional integrable Calogero-Bogoyavlenskii-Schiff equation. They obtained the solution of $(2+1)$-dimensional CBS equation;

$$
u(x, y, t)=3 k \operatorname{sech}^{2}\left(\sqrt{\frac{3 k}{2}}(x+a(y, t))\right)+k_{1}
$$

Where $a=a(y, t)$, satisfies $a_{t}=\emptyset(a) a_{z}$ and $k_{1}$ is an arbitrary constant. Here the similarity variable $x+a(y, t)$ connects y to t, while in our results in (31) and (32), contains the $\mathrm{d}$ 'Alembert form for $\mathrm{x}, \mathrm{t}$ and arbitrary functions giving many soliton shapes. Variety of exact solutions [21-24] of Calogero-Bogoyavlenskii-Schiff equation are constructed by using the improved $\left(\mathrm{G}^{\prime} / \mathrm{G}\right)$ expansion method. Family of exact solutions of CBS equation are obtained. The exact solution take the solitary wave form [21], when $A<0$

$$
\begin{aligned}
& u(\xi)=\alpha_{0}+2 \sqrt{-A} \tanh (\sqrt{-A} \xi)= \\
& \alpha_{0}+2 \sqrt{-A} \operatorname{coth}(\sqrt{-A} \xi)
\end{aligned}
$$

Where $\xi=x+z+4 A t, \alpha_{0}$ and $A$ are arbitrary constants The exact solution is for $\Delta_{1}=\frac{\sqrt{\lambda^{2}-4 \mu}}{2}$ [22];

$$
\begin{gathered}
u_{1}=-\frac{\left(-4 k^{2} \varphi_{y} \mu+k^{2} \varphi_{y} \lambda^{2}+\tau_{t}\right) x}{4 \varphi_{y}}+2 k\left(-\frac{\lambda}{2}+\right. \\
\Delta_{1}\left(\frac{C_{1} \cosh \left(\Delta_{1}(k x+\varphi+\tau)\right)+C_{2} \sinh \left(\Delta_{1}(k x+\varphi+\tau)\right)}{C_{1} \sinh \left(\Delta_{1}(k x+\varphi+\tau)\right)+C_{2} \cosh \left(\Delta_{1}(k x+\varphi+\tau)\right)}\right)
\end{gathered}
$$

The $\left(G^{\prime} / G\right)$ expansion method was used for $\lambda^{2}-4 \mu>[23]$ yields;

$$
\begin{gathered}
u(\xi)= \pm \frac{\sqrt{\lambda^{2}-4 \mu}}{2}\left(\frac{A_{1} \sinh \left(\frac{1}{2} \sqrt{\lambda^{2}-4 \mu}\right) \xi+A_{2} \cosh \left(\frac{1}{2} \sqrt{\lambda^{2}-4 \mu}\right) \xi}{A_{1} \cosh \left(\frac{1}{2} \sqrt{\lambda^{2}-4 \mu}\right) \xi+A_{2} \sinh \left(\frac{1}{2} \sqrt{\lambda^{2}-4 \mu}\right) \xi}\right) \\
v(\xi)=\frac{\lambda^{2}-4 \mu}{8}\left(\frac{A_{1} \sinh \left(\frac{1}{2} \sqrt{\lambda^{2}-4 \mu}\right) \xi+A_{2} \cosh \left(\frac{1}{2} \sqrt{\lambda^{2}-4 \mu}\right) \xi}{A_{1} \cosh \left(\frac{1}{2} \sqrt{\lambda^{2}-4 \mu}\right) \xi+A_{2} \sinh \left(\frac{1}{2} \sqrt{\lambda^{2}-4 \mu}\right) \xi}\right)^{2}
\end{gathered}
$$

The solution takes the form when $\lambda^{2}-4 \mu>0$ [24]

$$
\begin{gathered}
u_{1}=-\frac{3}{2} f^{\prime 2}(y)\left(\frac{C_{1} \sinh \left(\frac{1}{2} \sqrt{\lambda^{2}-4 \mu}\right) \xi+C_{2} \cosh \left(\frac{1}{2} \sqrt{\lambda^{2}-4 \mu}\right) \xi}{C_{1} \cosh \left(\frac{1}{2} \sqrt{\lambda^{2}-4 \mu}\right) \xi+C_{2} \sinh \left(\frac{1}{2} \sqrt{\lambda^{2}-4 \mu}\right) \xi}\right)^{2}+\frac{3}{2}\left(f^{\prime \prime}(y)-f^{\prime 2}(y) \lambda\right)\left(\frac{C_{1} \sinh \left(\frac{1}{2} \sqrt{\lambda^{2}-4 \mu}\right) \xi+C_{2} \cosh \left(\frac{1}{2} \sqrt{\lambda^{2}-4 \mu}\right) \xi}{C_{1} \cosh \left(\frac{1}{2} \sqrt{\lambda^{2}-4 \mu}\right) \xi+C_{2} \sinh \left(\frac{1}{2} \sqrt{\lambda^{2}-4 \mu}\right) \xi}\right)+ \\
\frac{1}{4}\left(-2 \mu f^{\prime 2}(y)-\lambda^{2} f^{\prime 2}(y)+3 \lambda f^{\prime \prime}(y)-f^{\prime \prime \prime}(y) / f^{\prime}(y)\right. \\
v_{1}=-\frac{3}{2} k f^{\prime}(y)\left(\frac{C_{1} \sinh \left(\frac{1}{2} \sqrt{\lambda^{2}-4 \mu}\right) \xi+C_{2} \cosh \left(\frac{1}{2} \sqrt{\lambda^{2}-4 \mu}\right) \xi}{C_{1} \cosh \left(\frac{1}{2} \sqrt{\lambda^{2}-4 \mu}\right) \xi+C_{2} \sinh \left(\frac{1}{2} \sqrt{\lambda^{2}-4 \mu}\right) \xi}-\right. \\
\frac{3}{2} k \lambda f^{\prime}(y)\left(\frac{C_{1} \sinh \left(\frac{1}{2} \sqrt{\lambda^{2}-4 \mu}\right) \xi+C_{2} \cosh \left(\frac{1}{2} \sqrt{\lambda^{2}-4 \mu}\right) \xi}{C_{1} \cosh \left(\frac{1}{2} \sqrt{\lambda^{2}-4 \mu}\right) \xi+C_{2} \sinh \left(\frac{1}{2} \sqrt{\lambda^{2}-4 \mu}\right) \xi}\right)-\frac{1}{4}\left(6 \mu k f^{\prime}(y)+g^{\prime}(t)\right)
\end{gathered}
$$

where $\xi=k(x)+f(y)+g(t), C_{1}$ and $C_{2}$ are arbitrary constants. Biao and Yong [25] obtained some exact analytical solutions, which contain soliton and periodic solutions to the generalized Calogero-Bogoyavlenskii-Schiff (GCBS) equation by using generalized Riccati equation expansion method and symbolic computations. They get the exact analytical solution of the GCBS equation;

$$
\begin{gathered}
u(x, y, t)=2 \sqrt{\frac{-y}{R_{t}}} R+F_{2}(t) \mp \sqrt{\frac{y}{t}} \tanh \{\sqrt{-R} \pm \\
{\left[\sqrt{-\frac{y}{4 R_{t}} x}-\ln \left(C_{1} \sqrt{\frac{y}{t}}+C_{2}\right)-(1+\alpha) \ln (y)+\alpha \ln (t)\right]}
\end{gathered}
$$

Alam and Tunc [26] applied the exponential function expansion method to construct exact solutions of the nonlinear Bogoyavlenskii equation. The solution was found when $\mu \neq 0, \lambda^{2}-4 \mu>0$ is

$$
\mathrm{u}(\mathrm{x}, \mathrm{y}, \mathrm{t})=\frac{1}{2} \lambda-\left(\frac{2 \mu}{\sqrt{\lambda^{2}-4 \mu} \tanh \left(\frac{\sqrt{\lambda^{2}-4 \mu}}{2}(\xi+E)\right)+\lambda}\right)
$$

Where $\xi=\mathrm{x}-\left(-\frac{1}{8} \lambda^{2}+\frac{1}{2} \mu\right) \mathrm{t}$ and $E$ is an arbitrary constant. Cesar and Gomez [27] used an improved tanh-coth method to obtain exact solutions of the Bogoyavlenskii equation. The exact solutions of the Bogoyavlenskii equation are

$u(x, y, t)=$
$\frac{1}{x+y+\frac{1}{32}\left(48 a_{1} a_{2}-32 k_{1}\right) t+\varepsilon_{0}}+\frac{a_{1} a_{2}\left(x+y+\frac{1}{32}\left(48 a_{1} a_{2}-32 k_{1}\right) t+\varepsilon_{0}\right)}{1-\sqrt{a_{1} a_{2}}\left(x+y+\frac{1}{32}\left(48 a_{1} a_{2}-32 k_{1}\right) t+\varepsilon_{0}\right)}$

$$
v(x, y, t)=\frac{u^{2}(x, y, t)}{2}-k_{1}
$$


with $\mathrm{a}_{1}, \mathrm{a}_{2}, \mathrm{k}_{1}$ and $\varepsilon_{0}$ arbitrary constants. The symmetry method has been carried over [28] to the Calogero Bogoyavlenskii Schiff equation to find exact solutions of this equation. The exact solution appears as the following

$$
u(x, y, t)=\frac{1}{2} y f(t)+A_{0}+\frac{2}{x-\int f(t) d t+y}
$$

Wazwaz [29] employed the Hirota's bilinear method to derive multiple-front solutions for the CalogeroBogoyavlenskii-Schiff equation. He obtained the solution of the CBS equation as following:

$$
u(x, y, t)=\frac{2 k_{1} e^{k_{1} x+m_{1} y-k_{1}^{2} m_{1} t}}{1+e^{k_{1} x+m_{1} y-k_{1}^{2} m_{1} t}}
$$

Saleh et al. [33] obtained exact solutions of CalgeroBogoyavlenskii- Schiff equation by using the singular manifold method after Lie reductions. They obtained the exact solutions of Calgero-Bogoyavlenskii- Schiff equation as:

$$
\begin{gathered}
\mathrm{u}(\mathrm{x}, \mathrm{y}, \mathrm{t})=\frac{2 \mathrm{c}_{3}\left(\sec \frac{\sqrt{\mathrm{c}_{1}}}{2}\left(\mathrm{x}-2 \sqrt{\mathrm{y}}-2 \sqrt{\mathrm{t}}+\mathrm{c}_{2}\right)\right)^{2}}{\frac{2 \mathrm{c}_{3}}{\sqrt{\mathrm{c}_{1}}} \tan \left(\frac{\sqrt{\mathrm{c}_{1}}}{2}\left(\mathrm{x}-2 \sqrt{\mathrm{y}}-2 \sqrt{\mathrm{t}}+\mathrm{c}_{2}\right)\right)+\mathrm{c}_{4}}-\sqrt{\mathrm{c}_{1}} \tan \frac{\sqrt{\mathrm{c}_{1}}}{2}(\mathrm{x}- \\
\left.2 \sqrt{\mathrm{y}}-2 \sqrt{\mathrm{t}}+\mathrm{c}_{2}\right)+\frac{\mathrm{c}_{1}}{6}(\mathrm{x}-2 \sqrt{\mathrm{y}}-2 \sqrt{\mathrm{t}})+\frac{\mathrm{c}_{1} \mathrm{c}_{2}}{6}+\frac{2(\mathrm{y}-1)}{\sqrt{\mathrm{t}}}(48)
\end{gathered}
$$

where, $c_{1}=4, c_{3}=1$ and $c_{2}=c_{4}=c_{5}=0$. Kumar [34] used the similarity transformations method via Lie-group theory to derive exact solutions of $(2+1)$-dimensional Calogero-Bogoyavlenskii-Schiff (CBS) equation. The result obtained shows a linear of $\mathrm{x}, \mathrm{y}$ terms weighted by $\mathrm{t}^{\varepsilon}$, where $\varepsilon=1-\mathrm{a}, \mathrm{a}-1$ or $\mathrm{t}-1$. The solution of CBS equation is

$$
\begin{gathered}
\mathrm{u}(\mathrm{x}, \mathrm{y}, \mathrm{t})=\frac{\mathrm{t}^{(\mathrm{t}-1) / 2}}{\mathrm{C}_{2}}+\frac{\mathrm{C}}{\mathrm{t}^{(1-\mathrm{a}) / 2}}+\frac{\mathrm{B}(1-\mathrm{a})}{\mathrm{t}^{(1+\mathrm{a}) / 2}} \mathrm{y} \\
\left(x t^{(a-1) / 2}-2 A \frac{t^{(a-1) / 2}}{C_{2}(a-1)}-4 B+B_{1}\right)+\frac{y}{4 t}+\int \frac{F_{2}(t)}{t^{(a+1) / 2}} d t
\end{gathered}
$$

Gandarias and Bruzon [35] obtained the solution of the (2+1)-dimensional integrable CBS equation by using classical Lie symmetries and travelling-wave reductions with variable velocity depending on the form of an arbitrary function. The solution of (CBS) equation

$$
u(x, y, t)=\sqrt{2} \tanh \left(\frac{x-f(y-\lambda t)}{\sqrt{2}}\right)
$$

where $\lambda=\frac{1}{2}, f(y-\lambda t)=y-\frac{t}{2}, \mathrm{t}=0.1$.

Some of the previous obtained results are hereafter plotted.

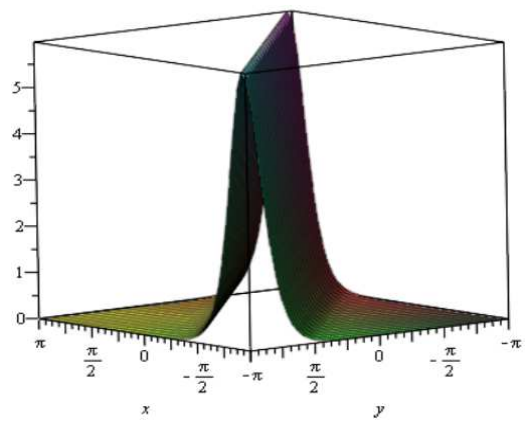

(a) $u=6 \operatorname{sech}^{2}(\sqrt{3}(x+y-t))$

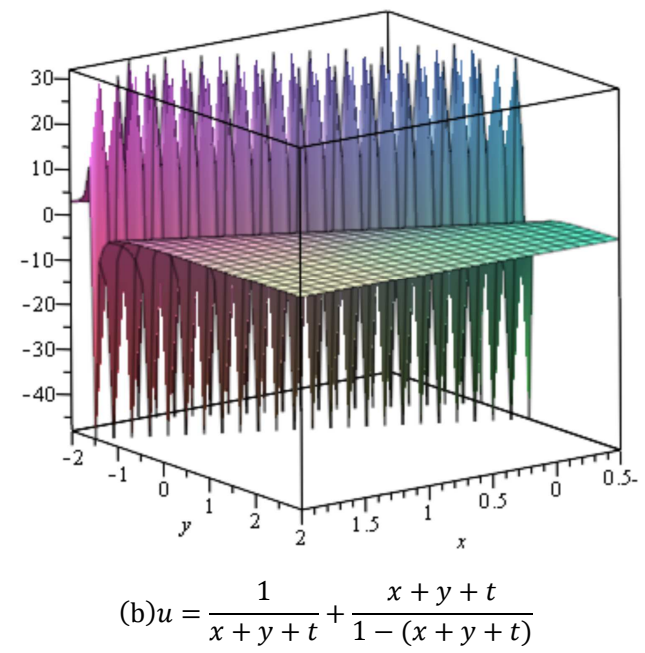

Figure 5. The soliton solution of Bruzon and Gandarias [20] and periodic solution of Cesar and Gomez [27].
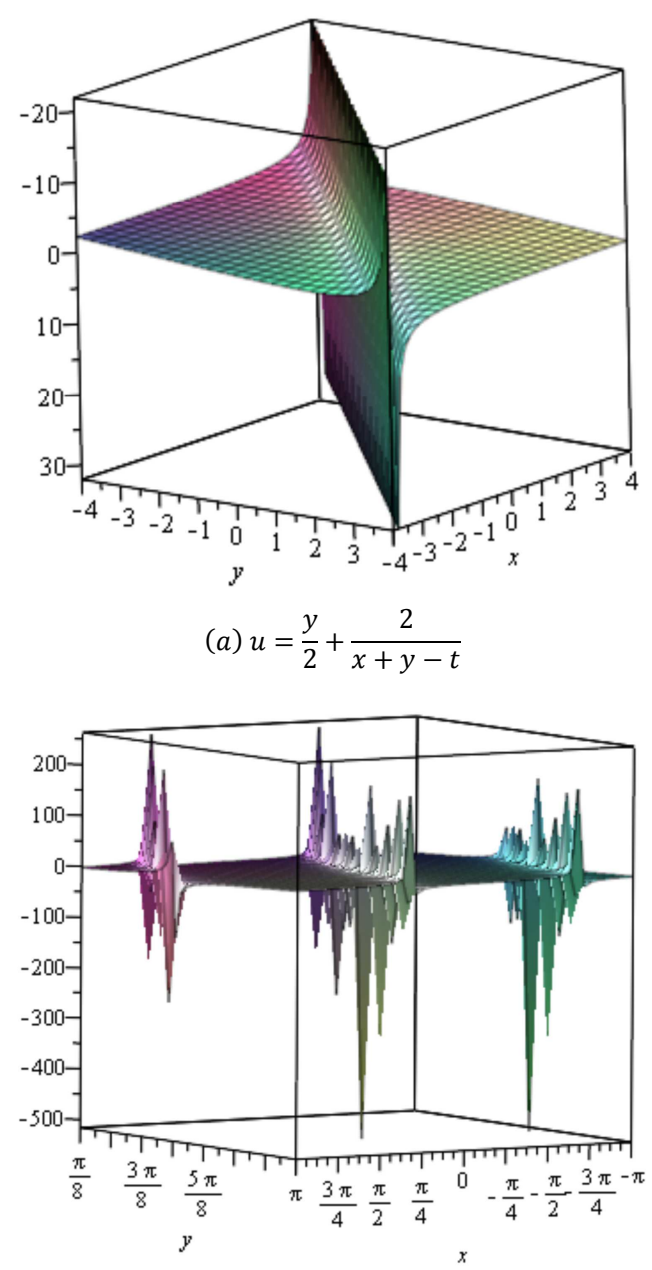

$$
\text { (b) } \begin{array}{r}
u=\frac{2(\sec (x-2 \sqrt{y}-2 \sqrt{t}))^{2}}{\tan ((x-2 \sqrt{y}-2 \sqrt{t}))}-2 \tan (x-2 \sqrt{y}-2 \sqrt{t}) \\
+\frac{4}{6}(x-2 \sqrt{y}-2 \sqrt{t})+\frac{2(y-1)}{\sqrt{t}}
\end{array}
$$

Figure 6. CBS solutions of Moatimid et al. [28] and Saleh et al. [33] respectively. 


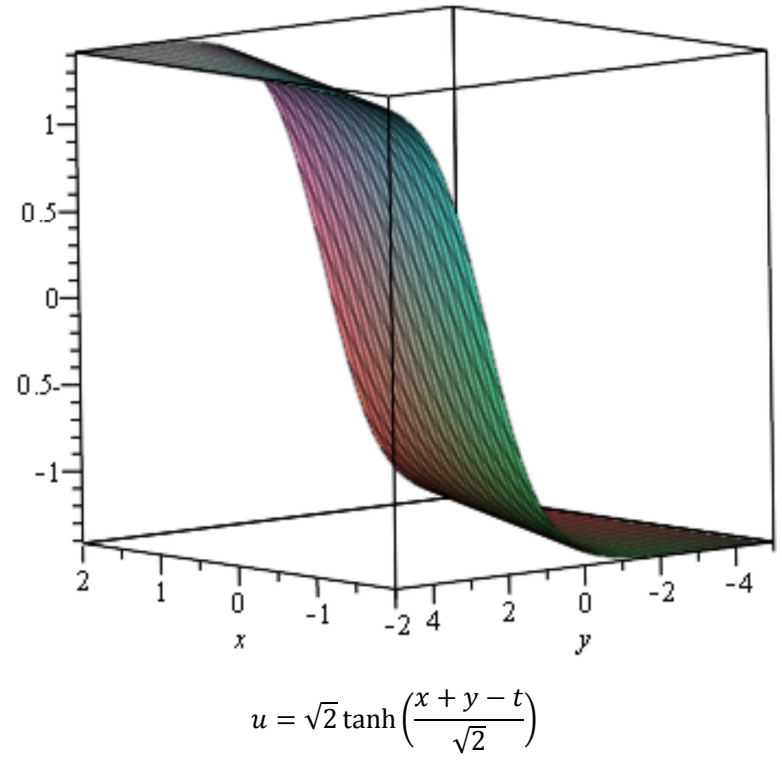

Figure 7. Solution of Gandarias and Bruzon [35].

It's clear from this comparison that we derive a new solution of Calogero-Bogoyavlenskii-Schiff equation by using a new method different from the previous findings.

\section{Conclusion}

Lax pair of (2+1) Calogero-Bogoyavlenskii-Schiff equation is obtained by using the singular manifold method. The detected Lie infinitesimals for the CBS Lax pair's contains eight unknown functions that are specialized by the aide of the commutator table. These functions are evaluated through the solution of a set of linear differential equations. Their solutions lead to optimal Lie vectors. The CBS Lax pair is reduced by using the optimal Lie vectors to a system of ODEs. New solutions for CBS equation are obtained and plotted for different arbitrary functions, reveal some solitary waves in the form of soliton and kink waves. The obtained solutions are compared with previous works. The comparison reveals that, the derived solutions are new and the detection of the Lax pair solution's is effective in exposure traveling wave solutions of nonlinear evolution equations.

\section{References}

[1] R. Hirota, The direct method in soliton theory, Cambridge University Press, 2004.

[2] J. Hietarinta, A search for bilinear equations passing Hirota's three-soliton condition. I. KdV-type bilinear equations, Journal of Mathematical Physics, 28 (1987) 1732-1742.

[3] J. P. Wang, A list of 1+1 dimensional integrable equations and their properties, Journal of Nonlinear Mathematical Physics, 9 (2002) 213-233.

[4] R. Yamilov, Symmetries as integrability criteria for differential difference equations, Journal of Physics A: Mathematical and General, 39 (2006) R541.
[5] S. M. Mabrouk, A. S. Rashed, Analysis of (3+1)-dimensional Boiti-Leon-Manna-Pempinelli equation via Lax pair investigation and group transformation method, Computers and Mathematics with Applications, 74 (2017) 2546-2556.

[6] R. Saleh, S. M. Mabrouk, M. Kassem, Truncation method with point transformation for exact solution of Liouville Bratu Gelfand equation, Computers and Mathematics with Applications, 76 (2018) 1219-1227.

[7] P. Estévez, J. Prada, Singular manifold method for an equation in $2+1$ dimensions, Journal of Nonlinear Mathematical Physics, 12 (2005) 266-279.

[8] S. M. Mabrouk, Chase-Repulsion analysis for (2+1)-Dimensional Lotka-Volterra System, International Journal of Engineering Research \& Technology, 8 (2019) 875-879.

[9] F. Engui, Z. Hong qing, L. Gang, Bäcklund transformation, lax pairs, symmetries and exact solutions for variable coefficient KdV equation, Acta Physica Sinica 7 (1998) 649.

[10] B. Cheng Lin, Extended homogeneous balance method and Lax pairs, Backlund transformation, Communications in Theoretical Physics, 37 (2002) 645.

[11] J. Ji, J. Wu, J. Zhang, Homogeneous balance method for an inhomogeneous KdV equation: Backlund transformation and Lax pair, Int. J. Nonlinear Sci, 9 (2010) 69-71.

[12] A. C. Newell, M. Tabor, Y. Zeng, A unified approach to Painlevé expansions, Physica D: Nonlinear Phenomena, 29 (1987) 1-68.

[13] H.-Q. Zhang, B. Tian, J. Li, T. Xu, Y.-X. Zhang, Symbolic-computation study of integrable properties for the $(2+1)$-dimensional Gardner equation with the two-singular manifold method, IMA journal of applied mathematics, 74 (2008) 46-61.

[14] M. Singh, Multi soliton solutions, bilinear Backlund transformation and Lax pair of nonlinear evolution equation in (2+1)-dimension, Computational Methods for Differential Equations, 3 (2015) 134-146.

[15] S.-J. Yu, K. Toda, T. Fukuyama, N-soliton solutions to a-dimensional integrable equation, Journal of Physics A: Mathematical and General, 31 (1998) 10181.

[16] K. Toda, Y. Song-Ju, T. Fukuyama, The Bogoyavlenskii-Schiff hierarchy and integrable equations in $(2+1)$ dimensions, Reports on Mathematical Physics, 44 (1999) 247-254.

[17] O. Bogoyavlenskiı̆, Overturning solitons in new two-dimensional integrable equations, Mathematics of the USSR-Izvestiya, 34 (1990) 245.

[18] A. M. Wazwaz, Multiple soliton solutions for the Bogoyavlenskii's generalized breaking soliton equations and its extension form, Applied Mathematics and Computation, 217 (2010) 4282-4288.

[19] A. M. Wazwaz, Integrable (2+1)-dimensional and $(3+1)$-dimensional breaking soliton equations, Physica Scripta, 81 (2010) 035005.

[20] M. Bruzon, M. Gandarias, C. Muriel, J. Ramirez, S. Saez, F. Romero, The Calogero-Bogoyavlenskii-Schiff equation in $2+1$ dimensions, Theoretical and mathematical physics, 137 (2003) 1367-1377. 
[21] M. Shakeel, S. T. Mohyud-Din, Improved $\left(\mathrm{G}^{\prime} / \mathrm{G}\right)$-expansion and extended tanh methods for (2+1)-dimensional CalogeroBogoyavlenskii-Schiff equation, Alexandria Engineering Journal, 54 (2015) 27-33.

[22] G. H. Xu, S. H. Ma, J. P. Fang, Investigation of $\left(\mathrm{G}^{\prime} / \mathrm{G}\right)$-Expansion Method and Exact Solutions for the (2+1)-Dimensional Calogero-Bogoyavlenskii-Schiff System, in: Applied Mechanics and Materials, Trans Tech Publ, 2013, pp. 235-239.

[23] A. Malik, F. Chand, H. Kumar, S. Mishra, Exact solutions of the Bogoyavlenskii equation using the multiple $\left(\mathrm{G}^{\prime}\right.$ G)-expansion method, Computers \& Mathematics with Applications, 64 (2012) 2850-2859.

[24] T. Xia, S. Xiong, Exact solutions of $(2+1)$-dimensional Bogoyavlenskii's breaking soliton equation with symbolic computation, Computers \& Mathematics with Applications, 60 (2010) 919-923.

[25] B. Li, Y. Chen, Exact analytical solutions of the generalized Calogero-Bogoyavlenskii-Schiff equation using symbolic computation, Czechoslovak journal of physics, 54 (2004) 517-528.

[26] M. N. Alam, C. Tunc, An analytical method for solving exact solutions of the nonlinear Bogoyavlenskii equation and the nonlinear diffusive predator-prey system, Alexandria Engineering Journal, 55 (2016) 1855-1865.

[27] A. Cesar, S. Gmez, Exact solution of the Bogoyavlenskii equation using the improved tanh-coth method, Nonlinear Dynamics, 70 (2015) 13-24.

[28] G. Moatimid, R. M. El-Shiekh, A.-G. A. Al-Nowehy, Exact solutions for Calogero-Bogoyavlenskii-Schiff equation using symmetry method, Applied Mathematics and Computation, 220 (2013) 455-462.

[29] A. M. Wazwaz, Multiple-soliton solutions for the CalogeroBogoyavlenskii-Schiff, Jimbo-Miwa and YTSF equations, Applied Mathematics and Computation, 203 (2008) 592-597.

[30] P. Estévez, P. Gordoa, The singular manifold method: Darboux transformations and nonclassical symmetries, Journal of Nonlinear Mathematical Physics, 2 (1995) 334-355.

[31] J. Weiss, The Painlevé property for partial differential equations. II: Bäcklund transformation, Lax pairs, and the Schwarzian derivative, Journal of Mathematical Physics, 24 (1983) 1405-1413.

[32] J. Weiss, M. Tabor, G. Carnevale, The Painlevé property for partial differential equations, Journal of Mathematical Physics, 24 (1983) 522-526.

[33] R. Saleh, M. Kassem, S. Mabrouk, Exact solutions of Calgero-Bogoyavlenskii-Schiff equation using the singular manifold method after Lie reductions, Mathematical Methods in the Applied Sciences, 40 (2017) 5851-5862.

[34] R. Kumar, Application of Lie-group theory for solving Calogero-Bogoyavlenskii-Schiff equation, IOSR J Math, 124 (2016) 144-147.

[35] M. Gandarias1, M. Bruzon1, Symmetry group analysis and similarity solutions of the CBS equation in $(2+1)$ dimensions, in: PAMM: Proceedings in Applied Mathematics and Mechanics, Wiley Online Library, 2008, pp. 10591-10592. 